\title{
The relationship between occlusal support and maxillary development: An animal study
}

\author{
Fabricio F. Da Costa ${ }^{1}$, Gabriela S. M. Q. Santos ${ }^{1}$, Arcelino Farias-Neto ${ }^{1}$, \\ Alfonso Sanchez-Ayala², Célia M. Rizzatti-Barbosa ${ }^{3}$
}

Correspondence: Dr. Arcelino Farias-Neto

Email: a.fariasneto@yahoo.com.br

\begin{abstract}
'Department of Dentistry, Health School, Potiguar University - Laureate International Universities, Natal, $\mathrm{RN}$, Brazil,

${ }^{2}$ Department of Dentistry, Ponta Grossa State University, School of Dentistry, Ponta Grossa, Paraná, Brazil, ${ }^{3}$ Department of Dentistry, Piracicaba Dental School, Campinas State University, Piracicaba, São Paulo, Brazil
\end{abstract}

\section{ABSTRACT}

Objective: To test the hypothesis that maxillary development may be affected by occlusal support. Materials and Methods: The sample was composed by Wistar rats ( 5 weeks old) divided into three groups: Control $(n=10)$, extraction of mandibular molar teeth - left side $(n=10)$, extraction mandibular molar teeth - left and right sides $(n=10)$. The rats were sacrificed 8 weeks postextraction. Cone beam computed tomography scan images were taken for posterior measurement of maxillary length and width. Data were analyzed by one-way analysis of variance (Tukey test as post-hoc test). Results: Maxillary length was significantly shorter $(P<0.005)$ in both groups after tooth extraction. No difference was observed regarding maxillary width and body weight. Conclusion: Reduced occlusal support may impair the development of the maxilla in rats.

Key words: Craniofacial growth, hypodontia, occlusal support

\section{INTRODUCTION}

Hypodontia is defined as the condition of having developmentally missing teeth, or it occurs when some of the teeth do not form and is also the most common anomaly in dental development. ${ }^{[1]}$ Hypodontia has been identified as both nonsyndromic when it is an independent congenital oral trait, or syndromic, when it is acquired as part of a specific disease and is usually of genetic origin. ${ }^{[1]}$ The prevalence of this condition has increased over the years in the human dentition as a general trend. It has a prevalence of 5.5\% in Europeans, with a preference for women compared to men (1.37:1)..$^{[2]}$

Loss of occlusal support has been related to different consequences. A topic of long-standing controversy is the relationship between loss of occlusal support, especially loss of molar support and the development of temporomandibular disorders. ${ }^{[3]}$ Jaw movements, bite force, and muscle activity may be affected when the occlusal support is below the normal value, which is 12-14 pairs of contacting teeth in an adult. ${ }^{[4]}$ Premature tooth loss often leads to space loss, alteration in the proper contact of the inclined planes of the teeth, and disturbance of masticatory function. ${ }^{[5]}$ Furthermore, premature loss of occlusal support may lead to masseter muscle atrophy ${ }^{[6]}$ and impairment in the development of the craniofacial structures. ${ }^{[7-9]}$

Possible reasons for a relationship between hypodontia and skeletal structures are, among others, the fact that teeth serve as functional units, whereby local bone growth is stimulated. ${ }^{[7]}$ Cross-sectional studies have shown an association between hypodontia and alterations in craniofacial structures. Kreczi et al.${ }^{[8]}$ examined the craniofacial structures of children with hypodontia in the maxilla, the mandible

\footnotetext{
How to cite this article: Da Costa FF, Santos GS, Farias-Neto A, Sanchez-Ayala A, Rizzatti-Barbosa CM. The relationship between occlusal support and maxillary development: An animal study. Eur J Dent 2015;9:400-3. 
or in both jaws. The authors observed bimaxillary retrognathism, reduction of the lower anterior facial height, and increased overbite and overjet when compared with subjects with complete dentition. ${ }^{[8]}$ Lateral cephalograms of 115 subjects with at least three congenitally missing teeth revealed the presence of more retruded maxillary and mandibular bones and a reduced Frankfort mandibular plane angle when compared with normal subjects. ${ }^{[9]}$ On the other hand, Bauer et al..$^{10]}$ examined the lateral cephalograms of 101 subjects prior to orthodontic treatment and found no significant correlation between craniofacial growth pattern and the congenital absence of certain permanent teeth. To the knowledge of the authors, no interventional study in animals has been found in the literature to clarify this relationship.

Since little consent about the influence of hypodontia on the development of craniofacial structures is found in literature, the purpose of this study was to evaluate the effect of premature loss of occlusal support on maxillary bone dimensions in growing rats. The research hypothesis is that premature loss of occlusal support may impair the development of the maxillary bone.

\section{MATERIALS AND METHODS}

The Ethics Committee on animal experiments of the institution approved the study. The sample was composed by female Wistar rats ( 5 weeks old) divided into three groups: Control $(n=10)$, extraction of mandibular molar teeth - left side $(n=10)$, extraction of mandibular molar teeth - left and right sides $(n=10)$. This sample size yielded a power of $80 \%$ for the primary outcome, maxillary length $(\alpha=0.05, \beta=0.20) .{ }^{[1]}$ Rats were provided with regular diet (normal rat pellets) and water ad libitum, at a constant temperature of $23^{\circ} \mathrm{C}$. Tooth extraction was performed on a surgical apparatus as previously described. ${ }^{[11]}$ Control rats were anesthetized and kept at maximum jaw opening for $10 \mathrm{~min}$ to simulate the surgical procedure [Figure 1]. Body weight was registered weekly during the study. The rats were sacrificed 8 weeks postextraction with an overdose of sodium pentobarbital (60 mg/kg; intraperitoneal injection). Heads were fixed in 10\% paraformaldehyde, and cone beam computed tomography scan images were taken. Maxillary length (right and left) were measured from the most anterior point of the maxilla in the frontal plane to the most distal point in the horizontal plane; maxillary width, as the greatest distance between the

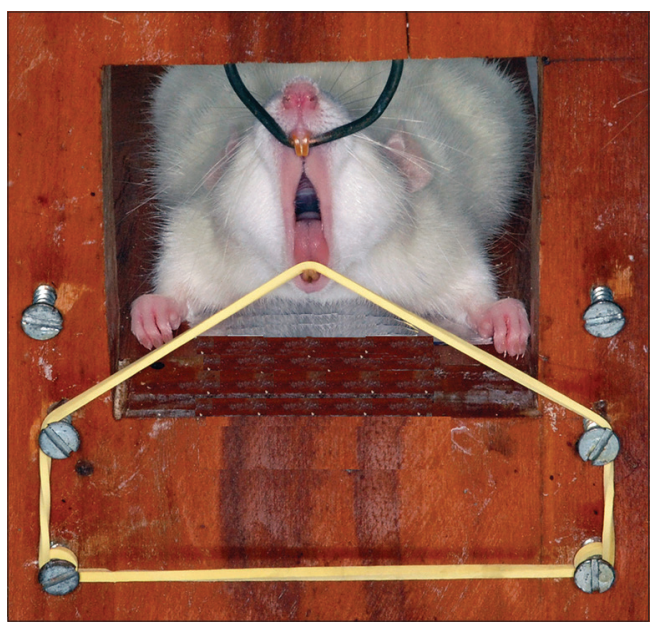

Figure 1: Control rats were anesthetized and kept at maximum jaw opening for $10 \mathrm{~min}$ to simulate the surgical procedure

lateral surfaces of the maxillary bone in the region of the second molar teeth [Figure 2]. Measurements were made by two independent observers at an interval of 4 weeks, and the averaged data were used to calculate the distances.

The data were processed with SPSS software (version 17.0 for Windows, SPSS Inc., Chicago, Ill, USA). The measurements of the two independent observers were submitted to the intra-class correlation test. The size of the method error in measuring the anatomical distances was calculated with the Dahlberg formula: $M E=\sqrt{\left[\sum \frac{d^{2}}{2 n}\right]}$, where $d$ is the difference between the two registrations of a pair, and $n$ is the number of double registrations. ${ }^{[12]}$ Ten mandibles were randomly selected for the evaluation of method error. The size of the method error in the measurements and the statistical significance between registrations are shown on Table 1.

Before carrying out the statistics, mandibular measurements were controlled for body size through the division of the linear measurements by the raw body weight. Maxillary length, maxillary width, and body weight were analyzed by one-way analysis of variance (Tukey test as post-hoc test). Shapiro-Wilk and Levene tests were used to observe normality and variance homogeneity, respectively. Confidence level was set at $5 \%$.

\section{RESULTS}

The intraclass correlation index $(\mathrm{ICC}=0.838, P<0.005)$ showed excellent reproducibility between the two observers. Table 2 shows the measurements of 
anatomical distances and body weight. Maxillary length was significantly shorter $(P<0.005)$ on both sides of the maxilla in the unilateral and bilateral extraction groups, but no difference was observed between sides in each group. No significant difference was observed regarding maxillary width and body weight.

\section{DISCUSSION}

The results of this study support de research hypothesis that premature loss of occlusal support may impair the development of the maxillary bone. The experimental animals exhibited a significantly shorter maxillary length at skeletal maturity. In a previous study with the same methodology, growing rats submitted to the extraction of molar teeth exhibited a smaller mandible at grown age. ${ }^{[11]}$ These results are interesting since they provide support for cross-sectional studies which observed retruded maxillary and mandibular bones in lateral cephalograms of subjects with hypodontia. ${ }^{[8,9]}$

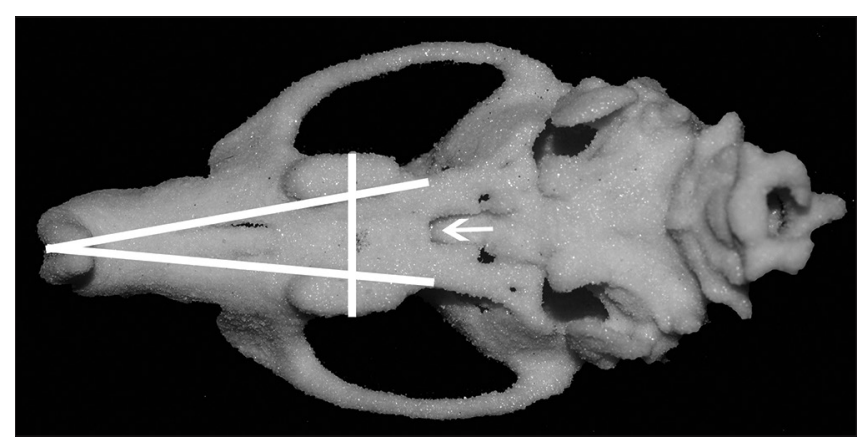

Figure 2: Maxillary length was measured as the most anterior point of the maxilla in the frontal plane to the most distal point in the horizontal plane. An imaginary line passing through the anatomic landmark indicated by the arrow was used to establish the posterior limit of the maxilla. Maxillary width was measured as the greatest distance between the lateral surfaces of the maxillary bone in the region of the second molar teeth
Results of previous cross-sectional studies on the effect of hypodontia on the development of craniofacial structures are not conclusive. While some studies observed retrognathic maxillary and mandibular bones, ${ }^{[8,9]}$ others observed more prognathic mandibles ${ }^{[12,13]}$ or no association between craniofacial growth and occlusal support. ${ }^{[10]}$ In the present study, a cause and effect relationship was observed. Rats had their mandibular molar teeth extracted at 5 weeks old and were followed up to 13 weeks, spanning the transition from early puberty to young adulthood. At 13 weeks old, skeletal maturity has been achieved, and rat bones continue growing at a reduced rate. ${ }^{[14]}$ Thus, animals were followed during a meaningful period of body development suitable for observation of bone morphologic changes. Animal growth was not compromised by nutrient intake, as evidenced by no significant difference for body weight among the groups. Further, maxillary measurements were controlled for body size before carrying out the statistics.

The possible link between hypodontia and skeletal structures may be the fact that teeth serve as functional units and stimulate local bone growth. It is supposed that after mandibular molar teeth extraction, muscle activity and force vectors may have been altered, leading to craniofacial growth impairment. For example, when the position of the mandible is altered as in mandibular advancement, force vectors are altered and mandibular growth enhanced. ${ }^{[15]}$ In a study with cadavers, a close relationship was found between loss of occlusal support and decreased masseter muscle volume and thickness, leading to masseter atrophy. ${ }^{[6]}$ Previous study in rats has shown that loss of occlusal support also leads to mandibular

Table 1: Size of method error in the ME and statistical significance between registrations

\begin{tabular}{lllll}
\hline Linear $\mathbf{M E}(\mathbf{m m})$ & ME & M (SD) I & M (SD) II & Difference $\boldsymbol{P}$ \\
\hline Maxillary length-right side & 0.14 & $21.39(0.51)$ & $21.19(0.41)$ & 0.34 \\
Maxillary length-left side & 0.11 & $21.45(0.47)$ & $21.29(0.44)$ & 0.45 \\
Maxillary width & 0.19 & $9.51(0.34)$ & $9.23(0.42)$ & 0.11 \\
\hline
\end{tabular}

ME: Measurements, M: Mean, SD: Standard deviation, registrations (I and II)

Table 2: ME of anatomical distances $(\mathrm{mm})$ and body weight $(\mathrm{g})$

\begin{tabular}{llccr}
\hline Study group & \multicolumn{3}{c}{ M (SD) } \\
\cline { 2 - 4 } & \multicolumn{2}{c}{ Maxillary length } & Maxillary width & Body weight \\
\cline { 2 - 4 } & $22.17(0.3)$ & $22.11(0.26)$ & $9.45(0.2)$ & $247.7(13.8)$ \\
\hline Control & $21.52^{*}(0.4)$ & $21.55^{*}(0.4)$ & $9.52(0.26)$ & $250.6(19.2)$ \\
Unilateral extraction & $21.2^{*}(0.35)$ & $21.27^{*}(0.35)$ & $9.43(0.3)$ & $250.9(13.7)$ \\
Bilateral extraction & *Significantly different in the same column at $P<0.05$. ME: Measurements, M: Mean, SD: Standard deviation &
\end{tabular}


growth impairment ${ }^{[11]}$ and affects the expression of proteins linked to bone metabolism (type II collagen, interleukin-1 $\beta$, and vascular endothelial growth factor) in the condylar cartilage, which is responsible for condylar growth. ${ }^{[16]}$ Muscle forces exert great influence on craniofacial growth and morphology. According to Moss's functional matrix theory, it is the investing soft tissues, especially the masticatory muscles, and the forces exerted by them that serve as the primary impetus for craniofacial growth and development. ${ }^{[7]}$

To summarize, premature loss of occlusal support in growing rats resulted in impairment on maxillary bone development. These results suggest that stable occlusion is necessary to achieve healthy craniofacial growth. Clinically, these results are very limited, but may shed some light on the link between hypodontia and the development of craniofacial structures.

\section{CONCLUSION}

Premature loss of posterior occlusal support impairs the development of the maxilla in growing rats.

\section{ACKNOWLEDGMENT}

This study was supported by the National Council for Scientific and Technological Development (CNPq), Ministry of Science and Technology, Brazil.

\section{REFERENCES}

1. Neville BW, Damm DD, Allen CM, Bouquot JE. Oral and Maxillofacial Pathology. $3^{\text {rd }}$ ed. Philadelphia: WB Sunders; 2009. p. 77-82.

2. Polder BJ, Van't Hof MA, Van der Linden FP, Kuijpers-Jagtman AM. A meta-analysis of the prevalence of dental agenesis of permanent teeth. Community Dent Oral Epidemiol 2004;32:217-26.

3. Haskin CL, Milam SB, Cameron IL. Pathogenesis of degenerative joint disease in the human temporomandibular joint. Crit Rev Oral Biol Med 1995;6:248-77.

4. Bakke M. Mandibular elevator muscles: Physiology, action, and effect of dental occlusion. Scand J Dent Res 1993;101:314-31.

5. Owen DG. The incidence and nature of space closure following the premature extraction of deciduous teeth: A literature study. Am J Orthod 1971;59:37-49.

6. Tetsuka M, Saga T, Nakamura M, Tabira Y, Kusukawa J, Yamaki K. Relationship between masseter muscle form and occlusal supports of remaining teeth. Kurume Med J 2012;59:5-15.

7. Moss ML. The primary role of functional matrices in facial growth. Am J Orthod Dentofacial Orthop 1969;55:556-65.

8. Kreczi A, Proff P, Reicheneder C, Faltermeier A. Effects of hypodontia on craniofacial structures and mandibular growth pattern. Head Face Med 2011;7:23.

9. Ben-Bassat Y, Brin I. Skeletodental patterns in patients with multiple congenitally missing teeth. Am J Orthod Dentofacial Orthop 2003;124:521-5.

10. Bauer N, Heckmann K, Sand A, Lisson JA. Craniofacial growth patterns in patients with congenitally missing permanent teeth. J Orofac Orthop 2009;70:139-51.

11. Farias-Neto A, Martins AP, Rizzatti-Barbosa CM. The effect of loss of occlusal support on mandibular morphology in growing rats. Angle Orthod 2012;82:242-6.

12. Nodal M, Kjaer I, Solow B. Craniofacial morphology in patients with multiple congenitally missing permanent teeth. Eur J Orthod 1994;16:104-9.

13. Woodworth DA, Sinclair PM, Alexander RG. Bilateral congenital absence of maxillary lateral incisors: A craniofacial and dental cast analysis. Am J Orthod 1985;87:280-93.

14. Roach HI, Mehta G, Oreffo RO, Clarke NM, Cooper C. Temporal analysis of rat growth plates: Cessation of growth with age despite presence of a physis. J Histochem Cytochem 2003;51:373-83.

15. Oksayan R, Sokucu O, Ucuncu N. Effects of bite-jumping appliances on mandibular advancement in growing rats: A radiographic study. Eur J Dent 2014;8:291-5.

16. Farias-Neto A, Martins AP, Sánchez-Ayala A, Rabie AB, Novaes PD, Rizzatti-Barbosa CM. The effect of posterior tooth loss on the expression of type II collagen, IL-1ß and VEGF in the condylar cartilage of growing rats. Arch Oral Biol 2012;57:1551-7.

\begin{tabular}{|l|l|}
\hline \multicolumn{2}{|c|}{ Access this article online } \\
\hline Quick Response Code: & Website: \\
\hline & www.eurjdent.com \\
\cline { 2 - 2 } & \\
\hline
\end{tabular}

